Aus dem Institut fur Tierzucht und Vererbungsforschung der Tierărztlichen Hochschule Hannover ${ }^{1}$ und dem Institut fur Tierpathologie, Ludwig-Maximilians-Universitat Monchen ${ }^{2}$

\author{
SUSANNE MÜLLER ${ }^{1,2}$, RÜDIGER WANKE ${ }^{2}$, WALTER HERMANNS ${ }^{2}$ \\ und OTTMAR DISTL'
}

\title{
Segregation von Pigmentzellanomalien bei Kreuzungen zwischen dem Münchener Miniaturschwein Troll und der Deutschen Landrasse
}

\author{
Herrn Professor Dr. D. Simon zum 70. Geburtstag gewidmet
}

\begin{abstract}
Summary
Title of the paper: Segregation of melanocytic lesions in crosses among the Munich Miniature Swine Troll and German Landrace

Since 1986, a line of Munich Miniature Swine (MMS) Troll showing a high incidence of spontaneous benign and malignant cutaneous melanocytic lesions has been established at the University of Munich. In order to study the inheritance of cutaneous melanocytic lesions in the Munich Miniature Swine Troll, we established the $\mathrm{F}_{1}$-,

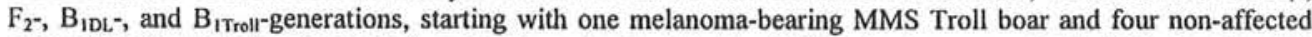
sows of the German Landrace (DL) as founder animals. A total of 176 animals were available, 27 in the $F_{1-}, 111$ in the $\mathrm{F}_{2^{-}}, 19$ in the $\mathrm{B}_{1 \mathrm{DL}}$, and 14 in the $\mathrm{B}_{1 \text { Troll }}$-generation. Benign melanocytic lesions with two distinct forms of basal melanocytic hyperplasia or nests of hyperplastic melanocytes like in human junctional nevus were observed in $10(41,7 \%) \mathrm{F}_{1-}^{-}, 20(18 \%) \mathrm{F}_{2}^{-}, 2(10,5 \%) \mathrm{B}_{\mathrm{IDL}}$, , and $7(50 \%) \mathrm{B}_{17 \text { roll-animals. Malignant melanomas }}$ were found in four $(3,6 \%) \mathrm{F}_{2}$ - and one $(7,1 \%) \mathrm{B}_{1 \text { Trolt }}$-animals, but did not occur in the $\mathrm{F}_{1}$ - and $\mathrm{B}_{1 \mathrm{DL}}$-generations. The observed segregation pattern suggests a different mode of inheritance for benign melanocytic lesions and melanomas, respectively. An influence of SLA haplotypes could not be observed. However, a significant influence of coat colour on the occurence of melanoma could be found in the $F_{2}$-generation. While around $65 \%$ of $\mathrm{F}_{2}$-animals had the German Landrace dominant white colour, melanomas were only found in black and red animals. Benign lesions of the junctional nevus type, too, were only found in black animals. A possible explanation is the lack of melanocytes in the skin of dominant white pigs caused by a mutation of the KIT-gene, which leads to a failure of melanoblast migration and development.
\end{abstract}

Key Words: Munich Miniature Swine Troll, melanoma, segregation, inheritance

\section{Zusammenfassung}

Am Institut für Tierpathologie der Tierärztlichen Fakultät München wird seit 1986 eine Sonderlinie des Münchener Miniaturschweins (MMS) Troll gezuchtet, die eine hohe Inzidenz sowohl benigner als auch maligner melanozyter Anomalien (Melanome bzw. năvoide Verănderungen) aufweist. Um Aufschlüsse uber den Vererbungsmodus dieser kutanen Pigmentzellanomalien zu gewinnen, wurden filr die vorliegende Arbeit, ausgehend von einem melanomtragenden MMS-Troll Eber und vier Sauen der Deutschen Landrasse, die $F_{1^{-}}, F_{2^{-}}$und reziproken Rückkreuzungsgenerationen erstellt. Insgesamt wurden 176 Tiere geboren, davon 27 in der $\mathrm{F}_{1}^{-}, 111$ in der $F_{2^{-}}, 19$ in der $R_{1 D L^{-}}$und 14 in der $R_{1 \text { troll- }}$ Generation. Benigne Lassionen in Form basaler Melanozytenhyperplasien zweier unterschiedlicher Grade sowie dem Junktionsnävus des Menschen vergleichbare Pigmentzellnester traten bei 10 Tieren $(41,7 \%)$ der $F_{1^{-}}, 20$ Tieren $(18,0 \%)$ der $F_{2^{-}}, 2$ Tieren $(10,5 \%)$ der $R_{1 D L}$ und 7 Tieren $(50 \%)$ der $R_{1 T \text { roll }}-$ Generation auf. Maligne Veränderungen (Melanome) wurden bei vier Tieren $(3,6 \%)$ der $F_{2}$ - und bei

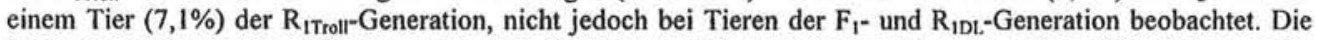
beobachtete Segregation läßt vermuten, daß fur benigne Pigmentzellanomalien ein anderer Vererbungsmodus wirksam ist als fur Melanome. Ein Einfluß des SLA- Haplotyps auf das Auftreten von Melanomen konnte nicht festgestellt werden. Ein signifikanter Einfluß auf das Auftreten von Melanomen konnte fur die Haut- und Haarfarbe der Tiere festgestellt werden. Obwohl etwa $65 \%$ der $\mathrm{F}_{2}$-Tiere die dominant weiße Fürbung der Deutschen Landrasse aufwiesen, traten Melanome nur bei schwarzen oder rot-schwarz gefleckten Tieren auf. Auch dem 
Junktionsnăvus des Menschen analoge Verănderungen traten nur bei schwarzen Tieren auf. Als mögliche Erklărung hierfür ist das Fehlen von Melanozyten in der Haut dominant weißer Schweine, das auf einer Mutation des KIT-Gens und einer daraus resultierenden Störung der Melanoblastenmigration und -entwicklung beruht, zu betrachten.

Schlüsselwörter: Münchener Miniatur Schwein Troll, Melanom, Segregation, Vererbung

1. Einleitung

Das Melanom des Menschen ist ein hochmaligner Tumor, der derzeit nur bei frühzeitiger Erkennung und Exzision gute Heilungschancen hat. Der in den letzten Jahrzehnten $\mathrm{zu}$ beobachtende deutliche Anstieg der Melanominzidenz bei Menschen weißer Hautfarbe hat zu einer Intensivierung der Melanomforschung geführt. Ein wichtiger Aspekt dabei sind die genetischen Grundlagen der Melanomentstehung. Es wird geschätzt, daß etwa $10 \%$ der Melanomfälle beim Menschen auf einer familiären Disposition beruhen. Ein an der Melanomentstehung in vielen Melanomfamilien beteiligtes Tumorsuppressor-Gen auf Chromosom 9 konnte bereits identifiziert werden. Es kodiert für das Protein p16, einen Inhibitor der cyclinabhängigen Kinase, die eine wichtige Rolle im Zellzyklus spielt. Dieser Tumorsuppressor weist auch bei zahlreichen sporadischen Melanomen und Tumoren anderer Gewebe Mutationen auf (KAMB et al., 1994). Es scheint sich beim familiären Melanom jedoch um ein genetisch heterogenes Merkmal zu handeln, denn in einem Teil der Fälle scheint kein Zusammenhang mit dem Protein p16, dafür aber mit Loci auf dem Chromosom 1 vorzuliegen (GOLDSTEIN et al., 1994). Auch Gene auf den Chromosomen 6, 7 und 11 scheinen in einem Teil der Fälle an der Melanomentstehung bzw. -entwicklung beteiligt zu sein (TRENT et al., 1990; BALABAN et al., 1986; TOMLINSON et al., 1993).

Beim Schwein treten Melanome im allgemeinen sehr selten auf. Eine Ausnahme hiervon bilden die Rassen Duroc, Sinclair Miniaturschwein und Münchener Miniaturschwein Troll (MMS-Troll). Die am Institut für Tierpathologie der Ludwig-Maximilians-Universität München gezüchtete Linie des MMS-Troll weist eine hohe Inzidenz sowohl benigner als auch maligner Pigmentzellanomalien auf, die eine eindeutig genetische Grundlage haben (WANKE und BRÄUER, 1986).

Die Segregation eines Merkmals läßt sich am besten in Kreuzungsgenerationen beobachten, die durch Anpaarung an eine für dieses Merkmal freie Linie oder Rasse erstellt werden. Zu diesem Zweck wurden aus der Anpaarung eines melanomtragenden MMS-Troll Ebers mit 4 Sauen der Deutschen Landrasse die $F_{1^{-}}, F_{2^{-}}$und reziproken Rückkreuzungsgenerationen erstellt. Die Untersuchungen sollten zeigen, welche Typen von Pigmentzellanomalien nach histologischer Klassifizierung in den einzelnen Generationen auftreten und ob sich signifikante Effekte auf die Verteilung der verschiedenen Klassen von Pigmentzellanomalien darstellen lassen.

Erstellung der $\mathrm{F}_{1}-\mathrm{F}_{2}$ - und $\mathrm{R}_{1}$-Generationen

Aus der Anpaarung eines melanomtragenden MMS-Troll Ebers mit 4 Sauen der Deutschen Landrasse (DL) ging die $\mathrm{F}_{1}$-Generation mit 24 Tieren hervor. Für die $\mathrm{F}_{2}$-Generation wurden die Tiere nach dem Schema befallen $\mathrm{x}$ befallen und nicht befallen $\mathrm{x}$ 
nicht befallen gepaart. Als Merkmal "befallen" dienten hier makroskopisch als benigne Pigmentzellanomalien anzusprechende schwarze Flecken auf der Rüsselscheibe, die bei einem Teil der Tiere auftraten. Für die Erstellung der $R_{1}-$ Generation wurden 2 befallene $F_{1}$-Eber auf die Mutterrasse DL sowie je eine befallene und eine nicht befallene $\mathrm{F}_{1}$-Sau auf den MMS-Troll Eber zurückgekreuzt.

Die Gesamtzahl der für die Untersuchung zur Verfügung stehenden Tiere betrug 176, wovon 5 der Elterngeneration, 27 der $F_{1-}, 111$ der $F_{2}$ - und 33 der $R_{1^{-}}$Generation angehörten (Tab. 1). Pathologisch-anatomisch untersucht wurden der Gründereber, $11 \mathrm{~F}_{1-\text {, }}$, $106 \mathrm{~F}_{2-}$ und $32 \mathrm{R}_{1}$-Tiere. Der Großteil der $\mathrm{F}_{2-}$ und $\mathrm{R}_{1}$-Tiere war zum Zeitpunkt der pathologisch-anatomischen Untersuchung zwischen 10 und 22 Wochen alt, die $F_{1}$ Tiere wurden bis zu einem Alter von 8 Monaten gehalten.

Tabelie 1

Materialubersicht (Survey on the material)

\begin{tabular}{lcccccc}
\hline & geboren & mănnlich & weiblich & aufgezogen & männlich & weiblich \\
\hline $\mathrm{F}_{1}$ & 27 & 13 & 14 & 24 & 12 & 12 \\
$\mathrm{~F}_{2}$ & 111 & 55 & 56 & 89 & 44 & 45 \\
$\mathrm{R}_{\text {I-DL }}$ & 19 & 11 & 8 & 17 & 9 & 8 \\
$\mathrm{R}_{\text {1-Troll }}$ & 14 & 6 & 8 & 8 & 4 & 4 \\
gesamt & 171 & 84 & 87 & 138 & 69 & 69 \\
\hline
\end{tabular}

\section{Datenerhebung}

Alle Ferkel wurden nach der Geburt klinisch untersucht. In einem Beobachtungsprotokoll wurden für jedes Tier Abstammung, Geburtsdatum, Geburtsgewicht, Wurfgröße und Farbe sowie Art, Lokalisation, Größe und Anzahl von vorhandenen Pigmentzellanomalien festgehalten. Außerdem wurde jedes Tier von beiden Seiten fotografiert und in 2- bis 4-wöchigen Abständen weiter beobachtet. Im Alter von 3-5 Monaten wurden die $\mathrm{F}_{2-}$ und $\mathrm{R}_{1}$-Tiere geschlachtet und anatomisch-pathologisch untersucht. Von pigmentierten Veränderungen der Haut, Lymphknoten und inneren Organe wurden Gewebeproben für die histologische Untersuchung entnommen. Außerdem wurden einigen nicht befallenen Tieren zur Kontrolle Proben von pigmentierter und unpigmentierter Haut entnommen. Totgeborene bzw. von der Sau erdrückte Ferkel wurden ebenfalls pathologisch-anatomisch untersucht.

Die Prüfung der Signifikanz der Häufigkeiten erfolgte mittels $\chi^{2}$-Testen in LogitModellen.

\section{Pathologisch-histologische Untersuchung}

Die bei der Sektion entnommenen Gewebeproben wurden $24 \mathrm{~h}$ in Formol (1:7) fixiert und anschließend in Paraffin eingebettet. Von diesen Paraffinblöcken wurden mit dem Schlittenmikrotom Schnitte von $1 \mu \mathrm{m}$ Dicke angefertigt. Diese wurden nach Strecken im Wasserbad auf einen Objektträger aufgezogen und über Nacht im Brutschrank bei $40{ }^{\circ} \mathrm{C}$ getrocknet. Danach erfolgte die Färbung der Präparate mit Hämalaun-Eosin (H.E.). Bei stark melaninhaltigen Präparaten wurde zur besseren Erkennbarkeit der Strukturen außerdem eine Bleichung mit anschließender H.E.-Färbung durchgeführt. 
Bei unklarer Diagnose wurde zusätzlich die Periodic Acid Schiff (PAS)-Reaktion und Giemsa-Färbung durchgeführt.

Die Bleichung der Schnitte erfolgte nach der von WEIDNER (1991) empfohlenen Methode nach Frangioni und Borgioli:

Die Schnitte werden 3 x 5 Minuten in Xylol entparaffiniert, in 100\%iges Äthanol und anschließend in die frisch zubereitete Bleichlösung gestellt. Diese Bleichlösung enthält

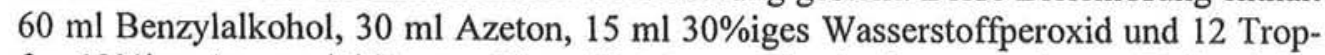
fen $12 \%$ ige Ammoniaklösung. Diese Zutaten werden in der angegebenen Reihenfolge gemischt, wobei nach jeder Zugabe mit einem Glasstab umgerührt wird. Die Schnitte werden in dieser Lösung in einem geschlossenen Glasgefäß bei $37^{\circ} \mathrm{C} 30$ bis $90 \mathrm{Minu}$ ten (je nach Pigmentierungsgrad) inkubiert. Danach spült man die Schnitte bei Raumtemperatur 10 Minuten lang in einer Azeton-Aqua dest.-Lösung (1:1) und anschließend 5 Minuten in reinem destilliertem Wasser. Um einen Teil des restlichen Melanins zu entfernen, stellt man die Schnitte für 10 Minuten in eine $20 \%$ ige Natriumsulfatlösung und spült sie anschließend mit Aqua dest.

\section{Ergebnisse}

Klinische und histologische Charakterisierung der beobachteten Pigmentzellanomalien

Bei der klinischen Untersuchung konnten zwei Arten von Pigmentzellanomalien unterschieden werden, tumoröse und nicht tumoröse. Die nicht tumorösen Formen stellten sich als schwarze, scharf abgegrenzte, nicht oder leicht erhabene Flecken mit unveränderter Hauttextur dar, deren Größe von wenigen Millimetern bis zu einem Durchmesser von $8 \mathrm{~cm}$ reichte. Bei der histologischen Untersuchung konnten diese nicht tumorösen Veränderungen in drei Typen eingeteilt werden:

Typ I: Bei diesem Typ handelte es sich um vorwiegend an der Rüsselscheibe weißer Tiere auftretende tiefschwarze, nicht erhabene Flecken. Histologisch waren sie durch eine orthotope, vorwiegend in den Spitzen der Reteleisten lokalisierte Melanozytenhyperplasie mit Hyperpigmentierung der basalen Zellschicht gekennzeichnet.

Typ II: Veränderungen dieses Typs traten vorwiegend am Rumpf, vereinzelt auch am Hals und den proximalen Extremitäten auf. Sie zeigten im histologischen Bild eine hochgradige lentigoide Melanozytenhyperplasie und hochgradige Hyperpigmentierung der Epidermis, vor allem der basalen Zellschicht. Vereinzelt war die Bildung basaler Melanozytennester und das Auftreten einzelner Melanozyten in den oberen Epidermisschichten zu beobachten.

Typ III: Bei diesem Typ war eine hochgradige Melanozytenhyperplasie mit deutlich erkennbarer Nesterbildung in der dermo-epidermalen Junktionszone zu beobachten. Dieser Typ entspricht dem Junktionsnävus des Menschen.

Tumoröse Pigmentzellanomalien stellten sich makroskopisch als schwarze, deutlich erhabene, gestielte oder kuppelförmige, häufig asymmetrische Knoten mit haarloser, schwarz glänzender oder rissiger Oberfläche dar mit einer Größe von bis zu 4,5 cm im Durchmesser. Eine Prädilektionsstelle war bei den Melanomen nicht festzustellen. Histologisch setzten sich diese Tumore aus zu Knoten zusammengelagerten, vorwiegend spindelförmigen, z. T. aber auch epitheloiden Melanomzellen und Anhäufungen pigmenthaltiger Makrophagen zusammen, die häufig die dermoepidermale Grenze 
auflösten und bis tief in die Dermis oder sogar bis in die Subkutis reichten. Die Epidermis war, soweit sie nicht durch Ulzeration zerstört war, verdickt und hyperkeratotisch und enthielt pagetoid verteilte Melanozyten. Neben dem MMS-Troll Gründereber wiesen fünf weitere Tiere Melanome auf, wovon vier Tiere aus der $\mathrm{F}_{2^{-}}$und ein Tier aus der $\mathrm{R}_{1 \text { Troll }}-$ Generation stammten. Bei vier dieser Tiere war bereits bei der Geburt ein Melanom vorhanden, bei dem fünten Tier entwickelte sich der Tumor in den ersten beiden Lebensmonaten. Bei einem der Tiere mit kongenitalem Melanom bildeten sich im Laufe der ersten drei Lebensmonate zwei weitere Melanome. Ein Teil der Tiere mit kutanem Melanom wies zusätzlich Metastasen in den regionären Lymphknoten oder inneren Organen und Melanosis verschiedener Organe auf. Bei den kutanen Melanomen des MMS-Troll Gründerebers war die Tumormasse vollständig zurückgebildet und hatte eine leicht über das Hautniveau erhabene, gelblich-weiße Narbe von derber Konsistenz zurückgelassen.

\section{Segregation der Pigmentzellanomalien}

Benigne Pigmentzellanomalien wurden in allen Generationen beobachtet. In der $\mathrm{F}_{1^{-}}$ Generation waren 10 von 24 Tieren davon betroffen, in der $F_{2}$-Generation 20 von 111 , in der $R_{1 D L}-$ Generation 2 von 19 und in der $R_{1 \text { Troll }}-$ Generation 7 von 14. Melanome

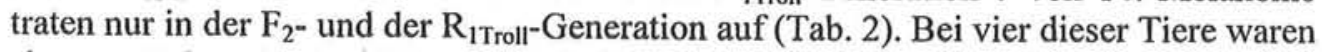
sie zum Zeitpunkt der Geburt vorhanden, bei einem Tier bildete sich der Tumor im Laufe des zweiten Lebensmonats. Nur bei einem Tier entstanden zusätzliche Melanome in den ersten drei Lebensmonaten.

In der $\mathrm{F}_{1}$-Generation wiesen in allen Würfen $50 \%$ oder mehr der Tiere benigne Pigmentzellanomalien auf, mit Ausnahme einer Anpaarung, wo nur eines von fünf Tieren betroffen war. In der $\mathrm{F}_{2}$-Generation war eine deutliche Erhöhung der Wurfgröße gegenüber der $\mathrm{F}_{1}$-Generation erkennbar. Sowohl benigne als auch maligne Pigmentzellanomalien traten hier unabhängig vom Befallsstatus der Eltern auf. Benigne Pigmentzellanomalien traten in allen Würfen auf, die vier Melanome waren auf vier verschiedene Elternpaare verteilt. Von den zwei Würfen aus der Rückkreuzung von $F_{1}$-Ebern auf DL-Sauen traten nur unter den Nachkommen des befallenen $F_{1}$-Ebers benigne Pigmentzellanomalien auf. Bei der Rückkreuzung von $\mathrm{F}_{1}$-Sauen auf den Gründereber

Tabelle 2

Ergebnisse der histologischen Untersuchung angeordnet nach Generationen (Results of the histological examination by generations)

\begin{tabular}{|c|c|c|c|c|c|c|c|c|c|}
\hline \multirow{2}{*}{$\begin{array}{l}\text { Gene- } \\
\text { ration }\end{array}$} & \multirow[t]{2}{*}{$\mathrm{n}$} & \multirow[t]{2}{*}{ o.b.B } & \multicolumn{4}{|c|}{ benigne PA } & \multicolumn{2}{|c|}{ Melanom } & \multirow[t]{2}{*}{ Melanosis } \\
\hline & & & $\begin{array}{c}\text { Typ } \\
\text { I }\end{array}$ & $\begin{array}{c}\text { Typ } \\
\text { II }\end{array}$ & $\begin{array}{l}\text { Typ } \\
\text { III }\end{array}$ & $\begin{array}{l}\text { ge- } \\
\text { samt }\end{array}$ & Haut & $\begin{array}{l}\text { Meta- } \\
\text { stasen }\end{array}$ & \\
\hline $\mathrm{P}$ (Eber) & 1 & & & & & & $1^{\circ}$ & 1 & 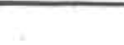 \\
\hline $\begin{array}{l}\mathrm{F}_{1} \\
\mathrm{~F}_{2} \\
\mathrm{R}_{1 \mathrm{DL}} \\
\mathrm{R}_{1 \text { Troll }}\end{array}$ & $\begin{array}{c}8 \\
34 \\
2 \\
7\end{array}$ & $\begin{array}{c}4 \\
15 \\
- \\
-\end{array}$ & $\begin{array}{c}3 \\
11 \\
2 \\
1\end{array}$ & $\begin{array}{l}1 \\
2 \\
- \\
4\end{array}$ & $\begin{array}{l}- \\
6 \\
- \\
2\end{array}$ & $\begin{array}{c}4 \\
19 \\
2 \\
7\end{array}$ & $\begin{array}{l}- \\
4 \\
- \\
1\end{array}$ & $\begin{array}{l}5 \\
- \\
-\end{array}$ & $\begin{array}{l}9 \\
- \\
-\end{array}$ \\
\hline gesamt & 52 & 19 & 17 & 7 & 8 & 32 & 6 & 4 & 9 \\
\hline
\end{tabular}


traten in beiden Würfen benigne Pigmentzellanomalien und in einem Wurf ein Melanom auf.

In der $F_{1}$-Generation wurden die makroskopisch sichtbaren Veränderungen von vier Tieren histologisch untersucht, drei davon wurden dem Typ I, eine dem Typ II zugeordnet. In der $\mathrm{F}_{2}$-Generation entsprachen die histologisch untersuchten Veränderungen von 19 Tieren elfmal dem Typ I, zweimal dem Typ II und sechsmal dem Typ III. Die zwei betroffenen Tiere der $\mathrm{R}_{1 \mathrm{DL}}$-Generation wiesen beide Veränderungen vom Typ I

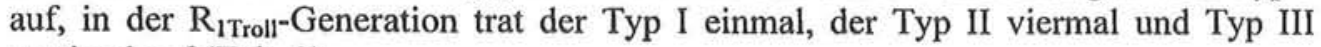
zweimal auf (Tab. 2).

\section{Einflüsse auf die Segregation der Pigmentzellanomalien}

Aus Tabelle 3 läßt sich ein signifikanter Generationseffekt für das Auftreten beider Arten von Pigmentzellanomalien erkennen. Während in der $F_{1}-$ Generation $41,7 \%$ der Tiere benigne Pigmentzellanomalien aufweisen, ist dies in der $\mathrm{F}_{2}$-Generation nur bei $18 \%$ der Tiere und in der $\mathrm{R}_{1 \mathrm{DL}}$-Generation nur bei $10,5 \%$ der Tiere der Fall. In der

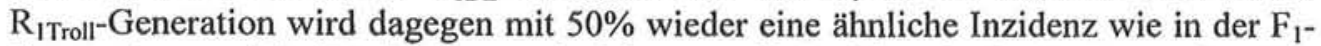
Generation erreicht. Für Melanome ist hingegen die Inzidenz in der $\mathrm{F}_{1}$-Generation $0 \%$, in der $\mathrm{F}_{2}$-Generation $3,6 \%$ und in der $\mathrm{R}_{1 \text { Troll }}$-Generation 7,1\%, während sie in der Rückkreuzung auf DL wiederum bei $0 \%$ liegt.

Tabelle 3

Inzidenz von Pigmentzellanomalien in den einzelnen Generationen (Incidence of melanocytic lesions in the different generations)

\begin{tabular}{l|c|c|c}
\hline Generation & Tierzahl & Nävi (\%) & Melanome (\%) \\
\hline $\mathrm{P}$ & 5 & 20,0 & 20 \\
\hline $\mathrm{F}_{1}$ & 24 & 41,7 & 0 \\
\hline $\mathrm{F}_{2}$ gesamt & 111 & 18,0 & 3,6 \\
männlich & 55 & 14,5 & 1,8 \\
weiblich & 56 & 21,4 & 5,4 \\
Eltern befallen & 49 & 20,4 & 2,0 \\
Eltern nicht befallen & 62 & 11,3 & 4,8 \\
weiß & 72 & 13,9 & 0 \\
nicht weiß & 39 & 18,0 & 10,3 \\
\hline $\mathrm{R}_{\text {1DL gesamt }}$ & 19 & 10,5 & 0 \\
\hline $\mathrm{R}_{\text {ITroll gesamt }}$ & 14 & 50,0 & 7,1 \\
\hline mit benignen Pigmentzellanomalien vom Typ I & & \\
\hline
\end{tabular}

Signifikante Effekte von Geschlecht, Befallsstatus der Eltern, Untersuchungsalter, Wurfgröße oder Wurfnummer für das Auftreten von benigner Pigmentzellanomalien und Melanomen konnten in der $\mathrm{F}_{2}$-Generation nicht festgestellt werden (Tab. 4). Auch ein Zusammenhang zwischen dem Befallsstatus und den Geburtsgewichten sowie täglichen Zunahmen konnte nicht ermittelt werden. Dagegen zeigte sich ein Zusammenhang zwischen der Haut- und Haarfarbe der Tiere und dem Auftreten von Melanomen. Das hier beobachtete alleinige Vorkommen von Melanomen bei Tieren mit nichtwei- 
Tabelle 4

Einflusse auf das Auftreten von Pigmentzellanomalien in der $\mathrm{F}_{2}$-Generation (Influences on occurrence of melanocytic lesions in the $\mathrm{F}_{2}$-generation)

\begin{tabular}{|c|c|c|c|c|}
\hline \multirow[t]{2}{*}{ Einflußfaktor } & \multirow[t]{2}{*}{ Faktorstufen } & \multicolumn{3}{|c|}{ Tiere } \\
\hline & & Anzahl & mit benignen PA (\%) & mit Melanom (\%) \\
\hline Wurfgröße & $\begin{array}{c}8 \\
11 \\
12 \\
15\end{array}$ & $\begin{array}{l}16 \\
44 \\
36 \\
15\end{array}$ & $\begin{array}{l}18,8 \\
13,6 \\
11,1 \\
46,7\end{array}$ & $\begin{array}{l}6,3 \\
2,3 \\
2,8 \\
6,7\end{array}$ \\
\hline Wurfnummer & $\begin{array}{l}1 \\
2\end{array}$ & $\begin{array}{l}51 \\
60\end{array}$ & $\begin{array}{l}15,7 \\
20,0\end{array}$ & $\begin{array}{l}2,0 \\
5,0\end{array}$ \\
\hline $\begin{array}{l}\text { Befallstatus } \\
\text { der Eltern }\end{array}$ & $\begin{array}{c}\text { befallen } \mathrm{x} \text { befallen }{ }^{1)} \\
\text { nicht befallen } \mathrm{x} \text { nicht } \\
\text { befallen }\end{array}$ & $\begin{array}{l}49 \\
62\end{array}$ & $\begin{array}{l}24,5 \\
12,9\end{array}$ & $\begin{array}{l}6,1 \\
1,6\end{array}$ \\
\hline Geschlecht & $\begin{array}{l}\mathrm{m} \\
\mathrm{w}\end{array}$ & $\begin{array}{l}55 \\
56\end{array}$ & $\begin{array}{l}14,6 \\
21,4\end{array}$ & $\begin{array}{l}1,8 \\
5,4\end{array}$ \\
\hline $\begin{array}{l}\text { Untersu- } \\
\text { chungsalter }\end{array}$ & $\begin{array}{l}\text { bis } 2 \text { Wochen } \\
6-10 \text { Wochen } \\
11-15 \text { Wochen } \\
16-20 \text { Wochen } \\
21-23 \text { Wochen }\end{array}$ & $\begin{array}{c}23 \\
6 \\
29 \\
33 \\
19\end{array}$ & $\begin{array}{c}4,4 \\
16,7 \\
24,1 \\
24,2 \\
15,8\end{array}$ & $\begin{array}{c}0,0 \\
16,7 \\
3,5 \\
3,0 \\
5,3\end{array}$ \\
\hline Haarfarbe & $\begin{array}{c}\text { weiß } \\
\text { nicht weiß }\end{array}$ & $\begin{array}{l}72 \\
39\end{array}$ & $\begin{array}{l}13,9 \\
25,6\end{array}$ & $\begin{array}{c}0,0 \\
10,3^{*}\end{array}$ \\
\hline Hautfarbe & $\begin{array}{c}\text { ganz weiß } \\
\text { dunkel pigmentiert }\end{array}$ & $\begin{array}{l}27 \\
30\end{array}$ & $\begin{array}{c}11,1 \\
33,3^{*}\end{array}$ & $\begin{array}{c}0,0 \\
10,0^{*}\end{array}$ \\
\hline
\end{tabular}

Differenz: $p<0,05$

ßer Haarfarbe ist signifikant für $\mathrm{p}<0,05$. Außerdem ist eine signifikante Häufung von benignen Pigmentzellanomalien, und hier vor allem vom Typ II und Typ III, bei Tieren mit schwarzer Haut- und Haarfarbe zu beobachten (Tab. 4). Ein Einfluß des SLAHaplotyps auf das Auftreten von Melanomen und Nävi war nicht zu erkennen (Tab. 5).

\section{Diskussion}

Ziel dieser Arbeit war es, Aufschlüsse über das Auftreten und die Erscheinungsform benigner und maligner Pigmentzellanomalien anhand der aus der Kreuzung eines melanomtragenden Ebers der Rasse Münchener Miniaturschwein Troll mit Sauen der Deutschen Landrasse hervorgegangenen $\mathrm{F}_{1^{-}}, \mathrm{F}_{2^{-}}$und reziproken Rückkreuzungsgenerationen zu gewinnen. Bei den Nachkommen aus der Kreuzung eines melanomtragenden MMS-Troll-Ebers mit Sauen der Deutschen Landrasse (DL) waren benigne und maligne Pigmentzellanomalien $\mathrm{zu}$ beobachten. Die benignen Pigmentzellanomalien konnten in drei verschiedene Typen unterteilt werden:

Die benignen Pigmentzellanomalien vom Typ II und Typ III sowie die malignen Tumore entsprechen den von WEIDNER (1991) für die am Institut für Tierpathologie der Tierärztlichen Fakultät München etablierte MMS-Troll Linie beschriebenen Veränderungen und den beim Sinclair Miniaturschwein (SMS) beobachteten Pigmentzellanomalien. Bisher nicht beobachtet wurde dagegen die benigne Pigmentzellanomalie vom 
Tabelle 5

Verteilung der SLA-Haplotypen in der $\mathrm{F}_{1}$ - und zu erwartende SLA-Haplotypen in der $\mathrm{F}_{2}$-Generation (Distribution of SLA haplotypes in the $\mathrm{F}_{1}$ - and SLA haplotypes being expected in the $\mathrm{F}_{2}$-generation)

\begin{tabular}{|c|c|c|c|c|}
\hline \multicolumn{2}{|c|}{ SLA-Haplotypen in der $F_{1}$} & $\begin{array}{l}\text { Anzahl der } \\
\text { Tiere }\end{array}$ & \multicolumn{2}{|c|}{$\begin{array}{l}\text { Inzidenz }(n) \text { von benignen } \\
\text { Pigmentzellanomalien }\end{array}$} \\
\hline \multicolumn{2}{|r|}{$04 / 04$} & 6 & \multicolumn{2}{|c|}{4} \\
\hline \multicolumn{2}{|r|}{$04 / 01$} & 14 & \multicolumn{2}{|r|}{5} \\
\hline \multicolumn{2}{|r|}{$04 / 14$} & 2 & \multicolumn{2}{|r|}{1} \\
\hline \multicolumn{2}{|r|}{$04 / 11$} & 1 & \multicolumn{2}{|r|}{1} \\
\hline $\begin{array}{l}\text { SLA der } F_{1-} \\
\text { Tiere }\end{array}$ & $\begin{array}{l}\text { SLA-Haplotypen } \\
\text { in der } F_{2}\end{array}$ & Anzahl Tiere & $\begin{array}{l}\text { Inzidenz (\%) } \\
\text { benignen PA }\end{array}$ & $\begin{array}{l}\text { von } \\
\text { Melanomen }\end{array}$ \\
\hline $\begin{array}{l}04 / 04 \\
04 / 04\end{array}$ & $04 / 04$ & 26 & 38,5 & 3,8 \\
\hline $\begin{array}{l}04 / 01 \\
04 / 04\end{array}$ & $\begin{array}{l}04 / 04 \\
04 / 01\end{array}$ & 23 & 8,7 & 4,3 \\
\hline $\begin{array}{l}04 / 14 \\
04 / 01\end{array}$ & $\begin{array}{l}04 / 04 \\
04 / 01 \\
14 / 04 \\
14 / 01\end{array}$ & 23 & 8,7 & 0,0 \\
\hline $\begin{array}{l}04 / 14 \\
04 / 04\end{array}$ & $\begin{array}{l}04 / 04 \\
14 / 04\end{array}$ & 20 & 15,0 & 5,0 \\
\hline $\begin{array}{l}04 / 14 \\
04 / 01\end{array}$ & $\begin{array}{l}04 / 04 \\
04 / 01 \\
14 / 04 \\
14 / 01\end{array}$ & 19 & 15,8 & 5,3 \\
\hline
\end{tabular}

Pigmentzellanomalien

Typ I. Obwohl es sich auch hier wie bei den Veränderungen vom Typ II um eine orthotope Pigmentzellhyperplasie handelt, unterscheidet sie sich von dieser durch den geringeren Grad der Melanozytenhyperplasie und der Hyperpigmentierung sowie durch das vorzugsweise Auftreten in den Spitzen der Reteleisten.

Die beobachteten Unterschiede in der Verteilung benigner und maligner Veränderungen zwischen der $F_{1}$ - und $R_{1 D L}$-Generation einerseits und der $F_{2}$ - und $R_{1 \text { Troll }}$-Generation zeigen, daß der Vererbung von Melanomen und benignen Pigmentzellanomalien unterschiedliche genetische Mechanismen zugrundeliegen. Das Auftreten von Typ ILäsionen in der $\mathrm{F}_{1}$-Generation läßt für diese Veränderung auf einen dominanten Erbgang schließen. Melanome sowie Nävi vom Typ II und III treten dagegen mit einer Ausnahme nur in der $\mathrm{F}_{2^{-}}$und $\mathrm{R}_{1 \text { Troll }}$-Generation auf. Für Melanome muß daher von einem rezessiven Erbgang ausgegangen werden, wobei ein Mehr-Locus-Modell der beobachteten Segregation deutlich besser angepaßt sein dürfte als das Ein-Locus-Modell. Dieses Ergebnis stimmt mit der Theorie von TISSOT et al. (1987) überein, die für die Vererbung von Melanomen beim Sinclair Miniaturschwein (SMS) von mindestens zwei nicht gekoppelten Loci ausgehen. Der von TISSOT et al. $(1987,1989,1993)$ bei Sinclair Miniaturschweinen festgestellte Einfluß von SLA-Haplotypen auf die Penetranz eines oder mehrerer Melanomgene(s) konnte in dem hier untersuchten Pedigree 
nicht festgestellt werden. Der MMS-Troll Gründereber wies wie alle anderen uns zur Verfügung stehenden MMS-Troll Schweine den SLA-Haplotyp H04/H04 auf. Trotz des alleinigen Vorkommens des Haplotyps H04 wird in der MMS-Troll Ursprungslinie eine hohe Frequenz von Melanomen gefunden. Die Hypothese von TISSOT et al. (1989, 1993), daß erst der SLA-Haplotyp B (vermutlich H10 nach der internationalen Nomenklatur) zur vollen Penetranz eines Tumor-Initiator-Gens führt, konnte damit für das MMS-Troll nicht bestätigt werden.

Es ist ein signifikanter Zusammenhang zwischen dem Auftreten der verschiedenen Pigmentzellanomalien und der Farbe der Tiere zu erkennen. Obwohl etwa $65 \%$ der $\mathrm{F}_{2}$ Tiere ganz oder vorwiegend weiß sind, treten in dieser Gruppe keine Melanome und auch keine benignen Läsionen vom Typ II oder Typ III auf, dagegen weisen von den restlichen $35 \%$, die nicht weiß sind, 10,3\% Melanome auf. Auch beim Sinclair Miniaturschwein waren bisher nur schwarze oder rote Tiere betroffen, die Signifikanz dieser Verteilung konnte jedoch aufgrund der geringen Zahl weißer Tiere in dieser Rasse nicht beurteilt werden (HOOK et al., 1979). In ihren Untersuchungen zum I-Locus stellten JOHANSSON MØLLER et al. (1995) fest, daß die Haarwurzeln und die Haut von Schweinen mit dominant weißer Farbe keine Melanozyten oder Vorläufer von Melanozyten enthält. Diese Beobachtung erhärtet die Hypothese, daß der I-Locus des Schweines mit dem KIT-Gen identisch ist. Für die Maus und den Menschen konnte nachgewiesen werden, daß Mutationen des KIT-Gens mit Störungen der Melanozytenmigration und -proliferation zusammenhängen. Ist eine solche Störung der Melanozytenmigration in die Haut die Grundlage der dominant weißen Farbe des Schweins, wäre das Fehlen von kutanen Melanomen bei weißen Schweinen in diesem Pedigree dadurch zu erklären. Denn selbst bei einer vorhandenen genetischen Prädisposition wäre die Expression kutaner Melanome bei vollständig weißen Tieren nicht möglich und bei weißen Tieren mit pigmentierten Hautpartien zumindest erheblich reduziert. Das würde bedeuten, daß es nur bei schwarzen und roten Tieren zur Expression von Melanomen kommt. Das Fehlen von Melanomen in der $F_{1}$ - und der $R_{1 D L}$-Generation könnte in diesem Fall auch damit erklärt werden, daß in diesen Generationen ausschließlich Tiere mit weißer Grundfarbe auftraten. Dieser möglicherweise vom I-Locus ausgehende Einfluß auf die Penetranz melanomprädisponierender Gene kann demnach auch für die überraschend niedrige $\mathrm{Zahl}$ befallener Tiere in der $\mathrm{F}_{2}$-Generation verantwortlich sein. Bezogen auf die Gesamtzahl der $\mathrm{F}_{2}$-Tiere liegt die Melanominzidenz bei $3,6 \%$, betrachtet man nur die Gruppe der schwarzen und roten Tiere, ergibt sich eine Inzidenz von $10,3 \%$.

\section{Literatur}

BALABAN, G.B.; HERLYN, M.; CLARK, W.H.; NOWELL, P.C.:

Karyotypic evolution in human malignant melanoma. Cancer Genet. Cytogenet. 19 (1986), 113-122 GOLDSTEIN, A.M.; DRACOPOLI, N.C.; ENGELSTEIN, M.; FRASER, M.C.; CLARK, W.H.; TUCKER
M.A.:

Linkage of cutaneous malignant melanoma/dysplastic nevi to chromosome $9 p$, and evidence for genetic heterogeneity. Am. J. Hum. Genet. 54 (1994), 489-496

HOOK, R.R.; AULTMAN, M.D.; ADELSTEIN, E.H.; OXENHANDLER, R.W.; MILIKAN, L.E.; MIDDLETON, C.C.:

Influence of selective breeding on the incidence of melanomas in Sinclair miniature swine. Int. J. Cancer 24 (1979), 668-672 
JOHANSSON MøLLER, M.; CHAUDHARY, R.; HELLMÉN, E.; HØYHEIM, B.; CHOWDHARY, B.P.; ANDERSON, L.:

Pigs with the dominant white coat colour phenotype carry a duplication of the KIT gene encoding the mast/stem cell growth factor receptor. In: MOLLER, M.: Comparative genome analysis in the pig. Univ. Uppsala, Schweden, Ph. Diss., 1995

KAMB, A.; GRUIS, N.A.; WEAVER-FELDHAUS, J.; LIU, Q.; HARSHMAN K.; TAVTIGIAN, S.V.;

STOCKERT, E.; DAY, R.S.; JOHNSON B.E.; SKOLNICK M.H.:

A cell cycle regulator potentially involved in genesis of many tumor types. Science, Washington 264 (1994), 436-440

TISSOT, R.G.; BEATTIE, C.W.; AMOSS, M.S.:

Inheritance of Sinclair swine cutaneous malignant melanoma. Cancer Res. 47 (1987), 5542-5545

TISSOT, R.G.; BEATTIE, C.W.; AMOSS, M.S.:

The swine leucocyte antigen (SLA) complex and Sinclair swine cutaneous malignant melanoma. Anim. Genet. 20 (1989), 51-57

TISSOT, R.G.; BEATTIE, C.W.; AMOSS, M.S.; WILLIAMS, J.D.; SCHUMACHER, J.:

Common leucocyte antigen (SLA) haplotypes in NIH and Sinclair miniature swine have similar effects on the expression of an inherited melanoma. Anim. Genet. 24 (1993), 191-193

TOMLINSON, I.P.M.; GAMMACK, A.J.; STICKLAND, J.E.; MANN, G.J.; MACKIE, R.M., KEFFORD,

R.F.; MCGEE, J.O.D.:

Loss of heterozygosity in malignant melanoma at loci on chromosomes 11 and 17 implicated in the pathogenesis of other cancers. Genes Chrom. Cancer 7 (1993), 169-172

TRENT, J.M.; STANBRIDGE, E.J.; MCBRIDE, H.L., MEESE, E.U.; CASEY, G.; ARAUJO, D.E., WITKOWSKI, C.M.; NAGLE, R.B.:

Tumorigenicity in human melanoma cell lines controlled by induction of human chromosome 6 . Science, Washington 247 (1990), 568-571

WANKE, R.; BRÄUER, H.:

Spontane Melanome in einer Sonderlinie des Münchener Miniaturschweins Troll. Berl. Münch. Tierärztl. Wschr. 99 (1986), 69

WEIDNER, C.:

Pigmentzellanomalien beim Münchener Miniaturschwein Troll - klinische, histochemische und enzymhistochemische Untersuchungen. Univ. München, Vet. med. Diss., 1991

Eingegangen: 03.09.1999

Akzeptiert: 12.01 .2000

Anschriften der Verfasser

Prof. Dr. OTTMAR DISTL, Dr. SUSANNE MÜLLER

Institut fur Tierzucht und Vererbungsforschung der Tierärztlichen Hochschule Hannover

Bünteweg 17p

D-30559 Hannover

E-Mail: odistl@zucht.tiho-hannover.de

PD Dr. RÜDIGER WANKE, Prof. Dr. WALTER HERMANNS

Institut für Tierpathologie, Ludwig-Maximilians-Universităt München

Veterinărstraße 13

D-80539 Munchen 